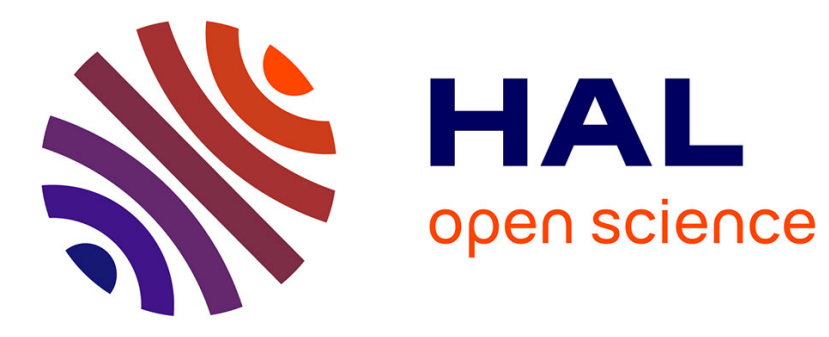

\title{
Low-power High Gain Observers for Wake Flow Rebuild
} Javeria Ahmed, Matthieu Fruchard, Estelle Courtial, Youssoufi Touré

\section{To cite this version:}

Javeria Ahmed, Matthieu Fruchard, Estelle Courtial, Youssoufi Touré. Low-power High Gain Observers for Wake Flow Rebuild. 2020 59th IEEE Conference on Decision and Control (CDC), Dec 2020, Jeju Island, South Korea. hal-03032884

\section{HAL Id: hal-03032884 https://hal.science/hal-03032884}

Submitted on 1 Dec 2020

HAL is a multi-disciplinary open access archive for the deposit and dissemination of scientific research documents, whether they are published or not. The documents may come from teaching and research institutions in France or abroad, or from public or private research centers.
L'archive ouverte pluridisciplinaire HAL, est destinée au dépôt et à la diffusion de documents scientifiques de niveau recherche, publiés ou non, émanant des établissements d'enseignement et de recherche français ou étrangers, des laboratoires publics ou privés. 


\title{
Low-power High Gain Observers for Wake Flow Rebuild
}

\author{
Javeria Ahmed, Matthieu Fruchard, Estelle Courtial and Youssoufi Touré
}

\begin{abstract}
This paper deals with the observation of the wake flow behind a bluff body based on reduced order models. Two Galerkin models with different truncation orders are considered to capture the intermittent character of the vortex shedding. The system output is the oscillation amplitude obtained thanks to pression measurements on the sides and on the back of the bluff body. For flow control purposes, the state estimation has to be efficient and robust to noises and dynamic changes. A high gain observer is modified i) by extending the state with the introduction of fictitious outputs in order to avoid an observability singularity and to ensure the existence of the inverse jacobian matrix and ii) by integrating a high gain parameter whose power is limited to 2 instead of $n$ to address the sensitivity issue of the standard high gain observer. Simulation results illustrate the efficiency of the proposed observers and highlight promising applications for flow control at high Reynolds numbers.
\end{abstract}

\section{INTRODUCTION}

\section{A. Context and motivation}

The flow at the rear of a bluff body is a common occurrence such as the flows at the back of an airplane, a submarine or a vehicle. The turbulent wakes downstream the bluff body can be very complex, exhibiting coherent structures with different scales. The strongly intermittent behavior of the flow depends on the Reynolds number. At high Reynolds numbers, vortex shedding occurs in the wake, leading to a significant pressure drop on the rear surface of the body. This phenomenon gives rise to structural vibrations, acoustic noise and increases the drag force. The control of vortex shedding is then of major interest for engineering applications. Active control in fluid mechanics is a promising and challenging research area. Promising because lower energy consumption and better performances are expected by decreasing the drag force. Challenging because observation and control require both real-time tractable and robust models with respect to dynamics changes in the wake flow [1], [2]. Fluid flows are governed by the Navier-Stokes equations known for their complexity (strong nonlinearities, high dimensionality and time-delays). This kind of modeling can not be used when real-time applications are targeted [3]. Linear models are unable to recover unstabilities characterizing the wake flow [4], [5]. Within this context, reduced order models approaches have been widely used to address the extraction of the coherent structures dynamics in fluid flows: Proper Orthogonal Decomposition [6] and more recently Dynamic

\footnotetext{
All the authors are with Laboratory PRISME, EA 4229, University of Orléans, INSA-CVL, 45072 Orléans, France. javeria.ahmed@etu.univ-orleans.fr, matthieu.fruchard, estelle.courtial, youssoufi.toure @univ-orleans.fr
}

Mode Decomposition [7], [8] using projection of the NavierStokes equations onto a modal basis. Galerkin models have proven to be a good compromise between simplicity and robustness. They are based on a Karhunen-Loève expansion around the steady or time-averaged Navier-Stokes solution $u_{s}$. The unsteady flow $u(x, t)$ is then approximated by an orthonormal Galerkin series:

$$
u(x, t) \approx u_{S}(x)+\sum_{i=1}^{2 N} a_{i}(t) u_{i}(x)
$$

where $u_{i}(x)$ denote the orthonormal spatial modes and $a_{i}(t)$ are the temporal modes amplitudes. For time periodic flows, structures are described by pairs of modes, acting as modal oscillators. Since the first two modes $(N=1)$ convey $95 \%$ of the turbulent kinetic energy, is it necessary to truncate the equation (1) at higher order? However, one mode plays a crucial role to robustify the low-order Galerkin model with respect to Reynolds range and transient dynamics [9], [10]: it's the shift mode, noted $a_{\Delta}$, modeling the energy flow between the mean flow and the vortex shedding. In the sequel, two Galerkin models are considered: a minimal Galerkin representation [9] using the dominant von Karman modes $a_{1}, a_{2}$ and the shift mode $a_{\Delta}$ (the decay rate $\mu>0$ is supposed to be constant):

$$
\left(\begin{array}{c}
\dot{a_{1}} \\
a_{2} \\
a_{\Delta} \\
\mu
\end{array}\right)=\left(\begin{array}{c}
\left(\mu-a_{\Delta}\right) a_{1}-a_{2} \\
\left(\mu-a_{\Delta}\right) a_{2}+a_{1} \\
a_{1}^{2}+a_{2}^{2}-a_{\Delta} \\
0
\end{array}\right)
$$

and a more refined Galerkin representation including higher damped $\left(\mu^{\prime}>0\right)$ modes $\left(a_{3}, a_{4}\right)$ interacting with the first two ones through the shift mode:

$$
\left(\begin{array}{c}
\dot{a_{1}} \\
a_{2} \\
a_{3} \\
a_{4} \\
a_{\Delta} \\
\mu
\end{array}\right)=\left(\begin{array}{c}
\left(\mu-a_{\Delta}\right) a_{1}-a_{2} \\
\left(\mu-a_{\Delta}\right) a_{2}+a_{1} \\
\left(-\mu^{\prime}-a_{\Delta}\right) a_{3}-a_{4} \\
\left(-\mu^{\prime}-a_{\Delta}\right) a_{4}+a_{3} \\
a_{1}^{2}+a_{2}^{2}+a_{3}^{2}+a_{4}^{2}-a_{\Delta} \\
0
\end{array}\right)
$$

The latter model, referred as a hybrid model [9], [11] between the empirical Galerkin model (2) and higher dimensional invariant-manifold model, is expected to be more precise. Considering the oscillation amplitudes $A_{i}=a_{2 i-1}^{2}+$ $a_{2 i}^{2}$, the above models become two systems of order 3 and 4 respectively. With the notation $x_{\text {min }}=\left(\begin{array}{lll}A_{1} & a_{\Delta} & \mu\end{array}\right) \in \mathscr{X} \subset$ $\mathbb{R}^{3}$, system (2) becomes:

$$
\mathscr{S}_{\text {min }}: \quad \dot{x}_{\text {min }}=\left(\begin{array}{c}
2 x_{1}\left(x_{3}-x_{2}\right) \\
x_{1}-x_{2} \\
0
\end{array}\right)=f\left(x_{\text {min }}\right)
$$


and using the state vector $x_{h y b}=\left(\begin{array}{llll}A_{1} & A_{2} & a_{\Delta} & \mu\end{array}\right) \in \mathscr{X} \subset$ $\mathbb{R}^{4}$, system (3) becomes

$$
\mathscr{S}_{h y b}: \quad \dot{x}_{h y b}=\left(\begin{array}{c}
2 x_{1}\left(x_{4}-x_{3}\right) \\
2 x_{2}\left(-\mu^{\prime}-x_{3}\right) \\
x_{1}+x_{2}-x_{3} \\
0
\end{array}\right)=f\left(x_{h y b}\right)
$$

For the sake of simplicity, the state vector $x$ will be used for both $x_{\min }$ and $x_{h y b}$ in the sequel. The only state component accessible to measurement is the oscillation amplitude $A_{1}$. So the output is $y=h(x)=x_{1}$ in both cases.

\section{B. Problem statement}

Rebuilding the unmeasured states is mandatory to $i$ ) understand the macro-physics at work in the wake, i.e. the dominant structure dynamics, and ii) synthesize control laws aiming at stabilizing the wake around some desired trajectory. A first prerequisite is to study the conditions guaranteeing the observability of the sytem.

Proposition 1 Systems $\mathscr{S}_{\min }$ and $\mathscr{S}_{\text {hyb }}$ respectively given by (4) and (5) are observable on $\Omega_{\min }=\left\{x \in \mathscr{X} \subset \mathbb{R}^{3}:\left|x_{1}\right|>\varepsilon\right\}$ and $\Omega_{h y b}=\left\{x \in \mathscr{X} \subset \mathbb{R}^{4}:\left|x_{1}\right|>\varepsilon,\left|x_{2}+x_{3}+\mu^{\prime}\right|>\varepsilon^{\prime}\right\}$ for any $\varepsilon, \varepsilon^{\prime}>0$, respectively.

Proof: Let $\mathscr{O}$ denote the smallest vector space containing $h$ and closed under the Lie derivative $\mathscr{L}_{f}$. A system is said weakly observable if $\operatorname{dim} \mathscr{O}(x)=\operatorname{dim} \mathscr{X}$ where $\mathrm{d} \mathscr{O}=\operatorname{Span}\{\mathrm{d} \tau, \tau \in \mathscr{O}\}$ is the observability co-distribution.

Computing the differentials of the successive Lie derivatives of the output $y=h(x)$ along the system vector field $f$ leads to the observability co-distributions $\mathrm{d} \mathscr{O}_{\min }$ and $\mathrm{d} \mathscr{O}_{\text {hyb }}$ for systems $\mathscr{S}_{\min }$ and $\mathscr{S}_{\text {hyb }}$ respectively, whose determinants are:

$$
\begin{aligned}
& \left|\mathrm{d} \mathscr{O}_{\text {min }}\right|=-4 x_{1}^{2} \\
& \left|\mathrm{~d} \mathscr{O}_{\text {hyb }}\right|=-16 x_{1}^{3}\left(x_{2}+x_{3}+\mu^{\prime}\right) .
\end{aligned}
$$

An observability singularity appears in particular when $x_{1}=$ 0 , whereas the control aims at forcing the oscillation amplitude to zero. The designed observer should be able to handle this singularity in order to synthesize control laws based on the estimated states.

Among the observer approaches, the High Gain Observer (HGO) is well-adapted to estimate nonlinear system states and exploits a fast error convergence depending on the high gain parameter. However, it has some flaws which need to be addressed properly. The power of its high gain parameter increases up to the dimension of the system causing some sensitivity issues. Besides, a non-singular diffeomorphism between the system expressed in natural coordinates and the system in its normal form is required.

\section{Contribution}

The present paper proposes a solution for the aforementioned issues. The first issue is addressed by limiting the power of the high gain parameter. As suggested by [12] for high dimensional physical systems, the proposed observer limits the high gain parameter power to two instead of $n$ at the expense of the increased observer dimension from $n$ to $2 n-2$. In order to deal with the singularity issue that happens during the inversion of the jacobian matrix to get back the observer in original coordinates, the HGO is modified by extending the state with the introduction of fictitious outputs [13]. In this way, we avoid the singularity by making the system observable and ensuring the existence of the inverse jacobian matrix everywhere in space $\mathbb{R}^{n}$.

This paper is organized as follows: we briefly recall the design of high gain observers in section 2 . Then the design of the proposed observer is detailed in section 3. Section 4 is devoted to the simulation results of the observation of the wake flow behind a bluff body. The observer efficiency for the two reduced order models are compared. Finally concluding remarks and on-going work are discussed in section 5 .

\section{Recall on High Gain Observers}

High gain observers [14], [15] are widely used since their arbitrarily fast exponential decay and practical stability arbitrary bound, in case of unmodeled dynamics, make possible to synthesize real-time output feedback stabilizing control laws for systems with few state measurements. We briefly recall the standard high gain observer, the low-power high gain observer and the observer in natural coordinates.

Proposition 2 (High gain observer) Consider the nonlinear system $\dot{x}=f(x), y=h(x)$ fulfilling the observability condition with $x \in \mathscr{X} \subset \mathbb{R}^{n}, y \in \mathbb{R}$. There exists an injective immersion $\phi$ from $\mathscr{X} \subset \mathbb{R}^{n}$ to $\mathbb{R}^{m}$ with $m \geq n$ such that the nonlinear system can be rewritten in the observability normal form with $z=\phi(x)$ as:

$$
\dot{z}=A_{m} z+B_{m} F(z), y=C_{m} z=z_{1}
$$

where the matrices $A_{m}, B_{m}$ and $C_{m}$ are in prime form: $A_{m}$ is $a(m \times m)$ square matrix whose non null entry is the unitary superdiagonal, $B_{m}\left(C_{m}^{T}\right)$ is a m-dimensional column vector whose non null entry is its unitary last (first) component. Then a high gain observer is given by

$$
\dot{\hat{z}}=A_{m} \hat{z}+B_{m} F_{s}(\hat{z})+\Delta_{m} K\left(y-C_{m} \hat{z}\right)
$$

with $\Delta_{m}=\operatorname{diag}\left(\theta, \ldots, \theta^{m}\right)$ where the high gain parameter $\theta>\theta_{0} \geq 1$ is related to the Lipschitz constant of $F_{s}, F_{s}$ is a saturated function of $F$ and $K$ is chosen such that the matrix $\left(A_{m}-K C_{m}\right)$ is Hurwitz.

Remark 1 Two well-known limitations of the high gain observer are related to the increasing powers of the high gain $\theta$ and to the inversion of $\phi$. First, as dimension increases, so does $\theta^{m}$, and it may result in an innovation term that dominates the observer dynamics and an increased sensitivity to noise on the last states, especially at high frequencies. Second, since the observer is built in the z-space, recovering an estimation useful for control purposes often requires to go back to the natural coordinates $x$, meaning that $\phi$ has to be inverted. This inversion is in general not easy, especially if $m>n$. 
In a recent study [12], the authors proposed a new observer of dimension $(2 m-2)$ using a high gain limited to power 2 instead of designing an observer of dimension $m$ with a gain that grows up to power $m$. The limited gain to power 2 reduces the numerical implementation problems of the classic high gain observer and also reduces the sensitivity to noise measurements.

Proposition 3 (Low-power high gain observer) Under assumptions and notations of Proposition 2, let

$$
\begin{aligned}
\dot{\zeta}_{i} & =A_{2} \zeta_{i}+N \zeta_{i+1}+\Delta_{2} K_{i} e_{i}, i \geq 1 \\
& \vdots \\
\dot{\zeta}_{m-1} & =A_{2} \zeta_{m-1}+B_{2} F_{s}(\zeta)+\Delta_{2} K_{m-1} e_{m-1}
\end{aligned}
$$

with $\zeta_{i} \in \mathbb{R}^{2}, \zeta_{0,2}=y$ given by convention, $e_{i}=\zeta_{i-1,2}-\zeta_{i, 1}$ and $K_{i}=\left(\begin{array}{ll}k_{i 1} & k_{i 2}\end{array}\right)^{T}$.

Let $N=\left(\begin{array}{ll}0_{2,1} & B_{2}\end{array}\right), \quad E_{i}=\left(\begin{array}{ll}-K_{i} & C_{2}^{T}\end{array}\right)$ and $Q_{i}=$ $\left(0_{2,1} \quad K_{i}\right)$. Choosing the high gain $\theta>\theta_{0}$ as in Proposition 2 and gains $K_{i}$ such that the matrix $M$

$$
M=\left(\begin{array}{ccccccc}
E_{1} & N & 0 & \ldots & & \ldots & 0 \\
Q_{2} & E_{2} & N & \ddots & & & \vdots \\
0 & \ddots & \ddots & \ddots & \ddots & & \vdots \\
\vdots & \ddots & Q_{i} & E_{i} & N & \ddots & \vdots \\
\vdots & & \ddots & \ddots & \ddots & \ddots & 0 \\
\vdots & & & \ddots & Q_{m-2} & E_{m-2} & N \\
0 & \ldots & & \ldots & 0 & Q_{m-1} & E_{m-1}
\end{array}\right)
$$

is Hurwitz then

$$
\dot{\zeta}=\Upsilon(\zeta, y)
$$

with $\Upsilon$ given by (9) provides a low-power high gain observer for system (7). We can use either $\hat{z}=P \zeta$ or $\hat{z}^{\prime}=P^{\prime} \zeta$ with the block diagonal projectors $P$ given by $I_{2}$ and $(m-1)$ matrices $B_{2}^{T}$, and $P^{\prime}$ given by $(m-1)$ matrices $C_{2}$ and $I_{2}$.

Remark 2 Despite this dimension increase, the low-power $\left(\Delta_{2}\right.$ depends only on $\left.\theta, \theta^{2}\right)$ high gain observer advantage is twofold: innovation term predominance is limited and sensitivity to output noise is consequently reduced, especially at high frequencies which is of particular interest considering wake flow dynamics.

Both aforementioned observers make use of a generically nonlinear mapping $\phi: x \mapsto z$ whose inversion is required to infer the observed state $\hat{x}$ in the original coordinates of the system dynamics. Such an inversion can be obtained through optimization but often at the price of costly computations. Furthermore, the non unicity of the inverse when $m>n$ may raise numerical issues. An alternative approach is to build the observer in the natural coordinates $x$ as suggested initially by [16].
Proposition 4 (Observer in natural coordinates) If the transformation $\phi: \mathscr{X} \subset \mathbb{R}^{n} \rightarrow \Pi \subset \mathbb{R}^{q}$ mapping the original system $\dot{x}=f(x)$ to a target system admitting an observer in the form $\dot{\pi}=\varphi(\pi, \hat{x}, y)$ is a diffeomorphism, then an observer in natural coordinates is given by:

$$
\dot{\hat{x}}=\frac{\partial \phi}{\partial x}(\hat{x})^{-1} \varphi(\phi(\hat{x}), \hat{x}, y) .
$$

Remark 3 Obviously, building an observer in natural coordinates is more difficult when $q=m>n$ using a high gain observer [17], or when $q=2(m-1)$ for low-power high gain observer, even if $m=n$.

\section{MAIN RESULTS}

To address the observability singularity avoidance, we follow the core idea initiated in [18] and then enhanced and structured in [13]. We follow a three steps procedure. The first step consists in augmenting the initial $n$ dimensional domain of the mapping $\phi$ to equal the dimension $q=$ $2(m-1)$ of the low-power observer. The resulting augmented mapping $\phi_{a}$ is now a diffeomorphism on a subset of $\mathbb{R}^{q}$. We then proceed to an extension of $\phi_{a}$ into an injective immersion $\phi_{e}$ on $\mathbb{R}^{q}$ using fictitious outputs to artificially remove the observability singularity. The third step lies in the augmentation of $\phi_{e}$ into a global diffeomorphism $\phi$ where we augment the natural coordinates by the coordinate $\tau \subset \mathbb{R}^{N-n}$ so that $\phi(x, 0)=\phi_{e}(x)$.

\section{A. A meaningful example}

Let us illustrate the previous section results as well as the observability singularity issue on the simple empirical Galerkin system (4). First we define $\phi \in \mathscr{C}^{1}\left(\Omega_{\text {min }} \subset \mathscr{X}, \mathbb{R}^{3}\right)$ using

$$
z=\phi(x)=\left(\begin{array}{c}
x_{1} \\
2 x_{1}\left(x_{3}-x_{2}\right) \\
2 x_{1}\left(2\left(x_{3}-x_{2}\right)^{2}-x_{1}+x_{2}\right)
\end{array}\right)
$$

so $\phi$ maps (4) on the observability canonical form (7) with $m=3$ and $F_{3}(x)=2 x_{1}\left[4\left(x_{3}-x_{2}\right)^{3}-2\left(x_{3}-x_{2}\right)\left(4 x_{1}-3 x_{2}\right)+\right.$ $\left.x_{1}-x_{2}\right]$. One can build an observer using Proposition 2 and get back to natural coordinates through the explicit expression of $\phi^{-1}$ :

$$
\hat{x}=\phi^{-1}(\hat{z})=\left(\begin{array}{c}
\hat{z}_{1} \\
\frac{\hat{z}_{1} \hat{z}_{3}-\hat{z}_{2}^{2}+2 \hat{z}_{1}^{3}}{2 \hat{z}_{1}^{2}} \\
\frac{\hat{z}_{1} \hat{z}_{2}+\hat{z}_{1} \hat{z}_{3}-\hat{z}_{2}^{2}+2 \hat{z}_{1}^{3}}{2 \hat{z}_{1}^{2}}
\end{array}\right)
$$

which is undefined at the singularity $\hat{z}_{1}=\hat{x}_{1}=0$. One can also get an observer in the natural coordinates using Propositions 2 and 4:

$$
\dot{\hat{x}}=\frac{\partial \phi}{\partial x}(\hat{x})^{-1}\left(A_{3} \phi(\hat{x})+B_{3} F_{s}(\hat{x})+\Delta_{3} K\left(y-C_{3} \hat{x}\right)\right)
$$

as long as $\phi$ is a diffeomorphism. Yet we know from Proposition 1 that $\phi$ is singular at $\hat{x}_{1}=0$ and there is no guarantee that the observer will not leave $\Omega_{\text {min }}$. 
Now using Propositions 2 and 3, we get a low-power high gain observer

$$
\dot{\zeta}=\varphi(\zeta, \hat{x}, y)=\left(\begin{array}{c}
\zeta_{12}+\theta k_{11}\left(y-\zeta_{11}\right) \\
\zeta_{22}+\theta^{2} k_{21}\left(y-\zeta_{11}\right) \\
\zeta_{22}+\theta k_{12}\left(\zeta_{12}-\zeta_{21}\right) \\
F_{3 s}(\zeta)+\theta^{2} k_{22}\left(\zeta_{12}-\zeta_{21}\right)
\end{array}\right)
$$

and we can get back to the natural coordinates using either $\hat{z}=P \zeta$ or $\hat{z}^{\prime}=P^{\prime} \zeta$ combined with (14). Note that such an observer exhibits the same redundancy as in using the lowpower high gain observer since we can use either $\hat{x}=P \hat{x}_{a}$ or $\hat{x}^{\prime}=P^{\prime} \hat{x}_{a}$. This observer also faces the problem linked with the observability singularity at $\hat{x}_{1}=0$.

\section{B. Augmentation of the mapping $\phi$}

Since the low-power high gain observer underlies a mapping $\phi$ from $x \in \mathbb{R}^{n}$ to $\zeta \in \mathbb{R}^{q}$ with $q=2(m-1)>m \geq n$, the mapping is not surjective for $m>2$. A first step is to augment the mapping into a diffeomorphism by augmenting the mapping domain. Since the state of the observer of Proposition 3 is redundant in the form (using $\hat{z}=P \zeta$ and $\left.\hat{z}^{\prime}=P^{\prime} \zeta\right)$

$\zeta=\left(\begin{array}{c}\zeta_{1} \\ \zeta_{2} \\ \vdots \\ \zeta_{n-2} \\ \zeta_{n-1}\end{array}\right)=\left(\begin{array}{c}\left(\begin{array}{l}\zeta_{1} \\ \zeta_{12} \\ \zeta_{2} \\ \zeta_{22}\end{array}\right) \\ \vdots \\ \left(\begin{array}{c}\zeta_{n-2} \\ \zeta_{n-2} \\ \zeta_{n-1} \\ \zeta_{n-1}\end{array}\right)\end{array}\right)=\left(\begin{array}{c}\left(\begin{array}{c}\hat{z}_{1} \\ \hat{z}_{2}\end{array}\right) \\ \left(\begin{array}{c}\hat{z}_{3}\end{array}\right) \\ \vdots \\ \left(\begin{array}{c} \\ \hat{z}_{n-1}\end{array}\right) \\ \left(\begin{array}{c}\hat{z}_{1}^{\prime} \\ \hat{z}_{n}\end{array}\right)\end{array}\right)=\left(\begin{array}{c}\left(\begin{array}{c}\hat{z}_{2}^{\prime} \\ \hat{z}^{\prime}\end{array}\right) \\ \vdots \\ \left(\begin{array}{c}\hat{z}_{n-2}^{\prime} \\ \hat{z}_{n-1}^{\prime} \\ \hat{z}_{n}^{\prime}\end{array}\right)\end{array}\right)$,

a natural way of addressing a diffeomorphism augmentation is to merge the two states $\hat{z}$ and $\hat{z}^{\prime}$ defining a new augmented state $\hat{z}_{a}=\left(\begin{array}{lllllll}\hat{z}_{1} & \hat{z}_{2} & \hat{z}_{2}^{\prime} & \hat{z}_{3} & \ldots & \hat{z}_{n-1}^{\prime} & \hat{z}_{n}\end{array}\right)^{T}$ associated with a natural coordinate augmented state $x_{a}=$ $\left(\begin{array}{lllllll}x_{1} & x_{2} & x_{2}^{\prime} & x_{3} & \ldots & x_{n-1}^{\prime} & x_{n}\end{array}\right)^{T} \in \mathbb{R}^{q}$.

Proposition 5 For systems $\mathscr{S}_{\min }$ and $\mathscr{S}_{\text {hyb }}$, defining respectively the natural coordinate augmented state $x_{a}$ and $\phi_{a}$ as

$$
\phi_{a}: x_{a}=\left(\begin{array}{c}
x_{1} \\
x_{2} \\
x_{2}^{\prime} \\
x_{3}
\end{array}\right) \mapsto z_{a}=\left(\begin{array}{c}
x_{1} \\
2 x_{1}\left(x_{3}-x_{2}\right) \\
2 x_{1}\left(x_{3}-x_{2}^{\prime}\right) \\
2 x_{1}\left(2\left(x_{3}-x_{2}\right)^{2}-x_{1}+x_{2}\right)
\end{array}\right)
$$

and $\phi_{a}: x_{a}=\left(\begin{array}{llllll}x_{1} & x_{2} & x_{2}^{\prime} & x_{3} & x_{3}^{\prime} & x_{4}\end{array}\right)^{T} \mapsto z_{a}$ given $b y$

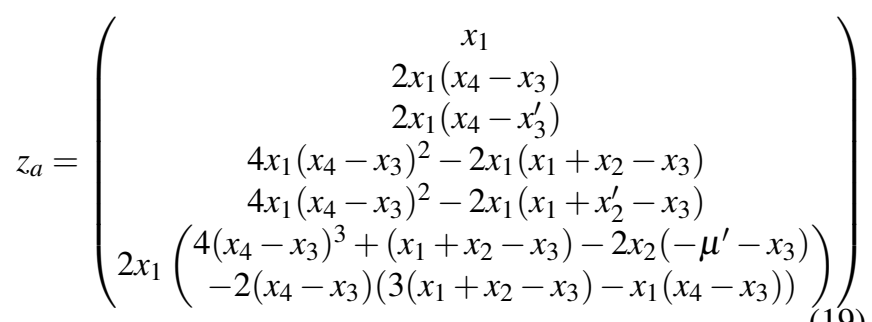

$\phi_{a}$ is a diffeomorphism on $\Omega_{a, \min }=\left\{x_{a} \in \mathbb{R}^{4}:\left|x_{1}\right|>\varepsilon\right\}$, respectively on $\Omega_{a, h y b}=\left\{x_{a} \in \mathbb{R}^{6}:\left|x_{1}\right|>\varepsilon,\left|x_{2}+x_{3}+\mu^{\prime}\right|>\right.$ $\left.\varepsilon^{\prime}\right\}$.

Proof: To lighten notations, one can write

$$
\frac{\partial \phi_{a}}{\partial x_{a}}\left(x_{a}\right)=\left(\begin{array}{cc}
1 & 0_{1, q-1} \\
* & J
\end{array}\right)
$$

For system $\mathscr{S}_{\min }$, differentiating $\phi_{a}$ leads to

$$
J_{\text {min }}=2\left(\begin{array}{ccc}
-x_{1} & 0 & x_{1} \\
0 & -x_{1} & x_{1} \\
x_{1}-4 x_{1} q_{0} & 0 & 4 x_{1} q_{0}
\end{array}\right)
$$

with $q_{0}=x_{3}-x_{2}$, so we have

$$
\left|\frac{\partial \phi_{a}}{\partial x_{a}}\left(x_{a}\right)\right|=x_{1}^{3}
$$

and $\phi_{a}$ is a diffeomorphism on $\Omega_{a, \min }$.

Similarly for the system $\mathscr{S}_{h y b}$, differentiating $\phi_{a}$ leads to

$$
J_{h y b}=2\left(\begin{array}{ccccc}
0 & 0 & -x_{1} & 0 & x_{1} \\
0 & 0 & 0 & -x_{1} & x_{1} \\
-x_{1} & 0 & -4 x_{1} q_{1}+x_{1} & 0 & 4 x_{1} q_{1} \\
0 & -x_{1} & -4 x_{1} q_{1}+x_{1} & 0 & 4 x_{1} q_{1} \\
x_{1} q_{2} & 0 & x_{1} q_{3} & 0 & x_{1} q_{4}
\end{array}\right)
$$

with $q_{1}=x_{4}-x_{3}, q_{2}=1-\left(-\mu^{\prime}-x_{3}\right)-6 q_{1}, q_{3}=-12 q_{1}^{2}+$ $6 q_{1}+8 x_{2}+8 x_{1}-6 x_{3}-1$ and $q_{4}=12 q_{1}^{2}-2\left(4 x_{1}+3 x_{2}-3 x_{3}\right)$. The determinant is given by

$$
\left|\frac{\partial \phi_{a}}{\partial x_{a}}\left(x_{a}\right)\right|=x_{1}^{5}\left(x_{2}+x_{3}+\mu^{\prime}\right),
$$

so $\phi_{a}$ is a diffeomorphism on $\Omega_{a, h y b}$.

Invertibility of the jacobian matrix of $\phi_{a}$ is now possible as long as the estimated augmented state remains away from the observability singularity. Yet there is no guarantee that the estimated state stays in this set. The next step addresses this concern.

\section{Extension of $\phi_{a}$ into an injective immersion on $\mathbb{R}^{q}$}

To avoid loosing any access to some unmeasured states because of the observability rank loss pinpointed in Proposition 1 , for instance the loss of information about $x_{2}$ and $x_{3}$ when $x_{1}=0$ for system $\mathscr{S}_{\min }$, a way to get around the observability singularity is to provide fictitious outputs aiming at getting information about these states around the singularity.

Proposition 6 For systems $\mathscr{S}_{\min }$ and $\mathscr{S}_{\text {hyb }}$, adding respectively fictitious outputs $y_{f} \in \mathbb{R}^{p}$

$$
y_{f}(x)=\left(\begin{array}{c}
\rho(x) x_{2} \\
\rho(x) x_{2}^{\prime} \\
\rho(x) x_{3}
\end{array}\right), \quad y_{f}(x)=\left(\begin{array}{c}
\rho(x) x_{2} \\
\rho(x) x_{2}^{\prime} \\
\rho(x) x_{3} \\
\rho(x) x_{3}^{\prime} \\
\rho(x) x_{4} \\
\rho^{\prime}(x)
\end{array}\right)
$$


, where $\rho$ and $\rho^{\prime}$ are differentiable functions that satisfy for all positive $\delta$ :

$$
\begin{array}{ll}
\rho\left(x_{1}\right): & \rho\left(x_{1}\right)+\delta x_{1}^{2}>0, \forall x_{1} \in \mathbb{R} \\
\rho^{\prime}\left(x_{2}, x_{3}\right): & \frac{\partial \rho^{\prime}}{\partial x_{2}}+\frac{\partial \rho^{\prime}}{\partial x_{3}}+x_{2}+x_{3}+\mu^{\prime}>0
\end{array}
$$

to the state $z_{a}$ results in an extended state $z_{e}=\left(\begin{array}{l}z_{a} \\ y_{f}\end{array}\right)$. Extending the transformation $\phi_{e}$ mapping $x_{a} \in \mathbb{R}^{q}$ to $z_{e} \in$ $\mathbb{R}^{q+p}$ results in an injective immersion on the domain $\mathbb{R}^{q}$.

Proof: The Jacobian matrix of $\phi_{e}$ is given by

$$
\frac{\partial \phi_{e}}{\partial x_{a}}\left(x_{a}\right)=\left(\begin{array}{cc}
1 & 0_{1, q-1} \\
* & J \\
* & J_{f}
\end{array}\right)
$$

where $J$ is $J_{\min }$ given by (21) ( $J_{h y b}$ given by (22)) for system $\mathscr{S}_{\text {min }}\left(\mathscr{S}_{\text {hyb }}\right)$ respectively, and $J_{f}$ is given by

$$
J_{f, \text { min }}=\rho I_{3}, \quad J_{f, h y b}=\left(\begin{array}{ccccc}
\multicolumn{7}{c}{} & \rho I_{5} & & \\
\rho_{2}^{\prime} & 0 & \rho_{3}^{\prime} & 0 & 0
\end{array}\right)
$$

where $\rho_{i}^{\prime}=\frac{\partial \rho^{\prime}}{\partial x_{i}}$. Since by construction $2 x_{1}^{2}+\rho(x)>0$ and $x_{2}+x_{3}+\mu^{\prime}+\rho_{2}^{\prime}(x)+\rho_{3}^{\prime}(x)>0$, it is straightforward that $\frac{\partial \phi_{e}}{\partial x_{e}}\left(x_{e}\right)$ has a constant rank $q$ for any $x_{a} \in \mathbb{R}^{q}$.

\section{Augmentation of $\phi_{e}$ into a global diffeomorphism}

The observability singularity has been solved, but using Proposition 4 requires a diffeomorphism to get an observer in natural coordinates. This last step consists in augmenting the immersion $\phi_{e}$ defined on the full domain $\mathbb{R}^{q}$ to $\mathbb{R}^{q+p}$ into a global diffeomorphism $\boldsymbol{\phi}$ on $\mathbb{R}^{q+p}$, which requires an augmentation of the natural coordinates $x_{a} \in \mathbb{R}^{q}$ to $\boldsymbol{x}=$ $\left(\begin{array}{c}x_{a} \\ \tau\end{array}\right) \in \mathbb{R}^{q+p}$. We follow here the Jacobian completion initially proposed in [18].

Proposition 7 Let $\tau \in \mathbb{R}^{p}$ and $\boldsymbol{x}=\left(\begin{array}{c}x_{a} \\ \tau\end{array}\right) \in \mathbb{R}^{q+p}$. Defining

$$
\boldsymbol{\phi}: \boldsymbol{x} \mapsto \boldsymbol{z}=z_{e}+\Psi\left(x_{a}\right) \tau=\phi_{e}\left(x_{a}\right)+\Psi\left(x_{a}\right) \tau
$$

with matrix $\Psi\left(x_{a}\right)$, the completion of the jacobian matrix $\frac{\partial \phi_{e}}{\partial x_{a}}$ :

$$
\frac{\partial \phi}{\partial \boldsymbol{x}}=\left(\begin{array}{ll}
\frac{\partial \phi_{e}}{\partial x_{a}}+\frac{\partial \Psi}{\partial x_{a}} & \Psi\left(x_{a}\right)
\end{array}\right)
$$

is fulfilled for systems $\mathscr{S}_{\min }$ and $\mathscr{S}_{\text {hyb }}$ using

$$
\Psi_{\min }\left(x_{a}\right)=\left(\begin{array}{c}
0_{1,3} \\
H_{1} \\
-B_{3}^{T} \\
x_{1} I_{3}
\end{array}\right) \text { with } H_{1}=\left(\begin{array}{ll}
I_{2} & -1_{2,1}
\end{array}\right)
$$

and

$$
\Psi_{h y b}\left(x_{a}\right)=\left(\begin{array}{cc}
0_{1,4} & 0 \\
H_{2} & 0_{4,1} \\
-B_{5}^{T} & -2 x_{1} \\
x_{1} I_{5} & 0_{5,1} \\
0_{1,4} & 1
\end{array}\right) \text { with } H_{2}=\left(\begin{array}{ccc}
0_{2} & I_{2} & -1_{2,1} \\
I_{2} & 0_{2} & -1_{2,1}
\end{array}\right) \text {. }
$$

Proof: Since $\Psi$ depends only on $x_{1}$, differentiating $\phi$ using (25) leads to

$$
\frac{\partial \boldsymbol{\phi}}{\partial \boldsymbol{x}}(\boldsymbol{x})=\left(\begin{array}{ccc}
1 & 0_{1, q-1} & \\
* & J & \Psi\left(x_{1}\right) \\
* & J_{f} &
\end{array}\right)
$$

Due to expressions (29) and (30), it follows that

$$
\begin{aligned}
& \left|\frac{\partial \boldsymbol{\phi}}{\partial \boldsymbol{x}}(\boldsymbol{x})\right|=\left(\rho+2 x_{1}^{2}\right)^{3} \\
& \left|\frac{\partial \boldsymbol{\phi}}{\partial \boldsymbol{x}}(\boldsymbol{x})\right|=\left(\rho+2 x_{1}^{2}\right)^{4}\left(2 x_{1}^{2}\left(\rho_{2}^{\prime}+\rho_{3}^{\prime}+2\left(x_{2}+x_{3}+\mu^{\prime}\right)\right)+\rho\right)
\end{aligned}
$$

for $\mathscr{S}_{\min }$ and $\mathscr{S}_{\text {hyb }}$, respectively. By construction of functions $\rho$ and $\rho^{\prime}$, these Jacobian determinants are strictly positive for all $\boldsymbol{x} \in \mathbb{R}^{q+p}$.

\section{E. Low-power high gain observer in natural coordinates}

Proposition 8 The dynamic system

$$
\dot{\hat{\boldsymbol{x}}}=\left(\frac{\partial \boldsymbol{\phi}}{\partial \boldsymbol{x}}(\hat{\boldsymbol{x}})\right)^{-1}\left(\begin{array}{c}
\Upsilon(\boldsymbol{\phi}(\hat{\boldsymbol{x}}), y) \\
-K_{p} \boldsymbol{\phi}_{p}(\hat{\boldsymbol{x}})
\end{array}\right)
$$

is a converging observer for system $\mathscr{S}_{\min }\left(\mathscr{S}_{\text {hyb }}\right)$ in their natural coordinates on any bounded subset of $\mathbb{R}^{q+p}$ with $(q, p)=(4,3)$ (with $(q, p)=(6,6)) . \Upsilon$ is defined by Proposition 3, $\phi$ is defined by Propositions 5, 6 and 7, $K_{p}$ is a positive definite diagonal matrix of dimension $p$ and the notation $\boldsymbol{\phi}_{p}$ stands for the last $p$ components of $\boldsymbol{\phi}$.

Proof: It can be checked that $\phi^{-1} \in \mathscr{C}^{1}$, hence it is $L$-Lipschitz on any compact of $\mathbb{R}^{q+p}$. It follows that, using $z^{*}=\boldsymbol{\phi}\left(x_{a}, 0\right)=\phi_{e}\left(x_{a}\right)$ :

$$
\begin{aligned}
\left\|x_{a}(t)-\hat{x}_{a}(t)\right\|+\|\tau(t)\| & \leq \sqrt{2}\left\|\begin{array}{c}
x_{a}-\hat{x}_{a} \| \\
\tau
\end{array}\right\| \\
& \leq \sqrt{2}\left\|\left(\begin{array}{c}
x_{a} \\
0
\end{array}\right)-\left(\begin{array}{c}
\hat{x}_{a} \\
\tau
\end{array}\right)\right\| \\
& \leq \sqrt{2}\left\|\boldsymbol{\phi}^{-1}\left(z^{*}\right)-\boldsymbol{\phi}^{-1}(\hat{z})\right\| \\
& \leq L \sqrt{2}\left\|z^{*}-\hat{z}\right\| \\
& \leq L \sqrt{2}\left\|\phi_{e}\left(x_{a}\right)-\boldsymbol{\phi}\left(\hat{x}_{a}, \tau\right)\right\| .
\end{aligned}
$$

Since $\phi_{e}$ is injective and (9) defines a converging observer from Proposition 3, it follows that

$$
\lim _{t \rightarrow \infty}\left\|x_{a}(t)-\hat{x}_{a}(t)\right\|+\|\hat{\tau}(t)\|=0 .
$$

Then $\boldsymbol{\phi}$ defines a global diffeomorphism.

\section{SIMULATION RESULTS}

\begin{tabular}{|c|cc|}
\hline$x_{0}$ & $\left(\begin{array}{lll}0 & 0.51 & 0.8\end{array}\right)^{T}$ \\
$\hat{x_{0}}$ & $\left(\begin{array}{ccc}0.1 & 0.1 & 0.2\end{array}\right)^{T}$ \\
$K_{1}, K_{2}$ & $\left(\begin{array}{c}0.18 \\
0.1\end{array}\right),\left(\begin{array}{l}0.18 \\
0.02\end{array}\right)$ \\
$K_{p}$ & \multicolumn{2}{|c}{$I_{3}$} \\
$\varepsilon$ & 0.5 \\
$\theta$ & 2 \\
\hline
\end{tabular}

TABLE I

INITIAL CONDITIONS AND PARAMETERS FOR SYSTEM $\mathscr{S}_{\text {min }}$ 


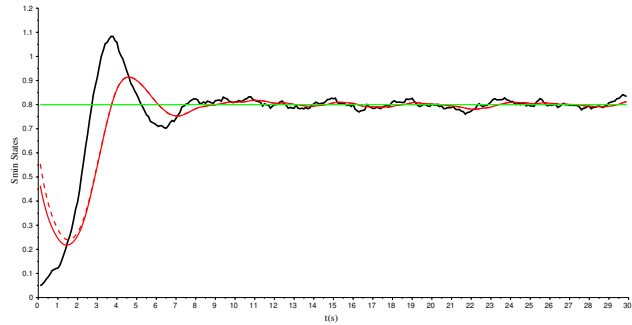

(a) $\mathscr{S}_{\text {min }}$ states

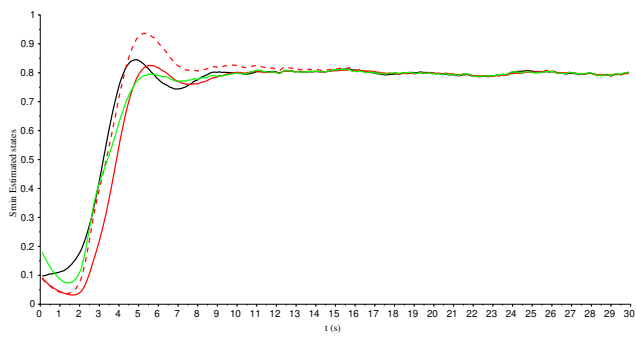

(c) Observer estimation for $\mathscr{S}_{\text {min }}$

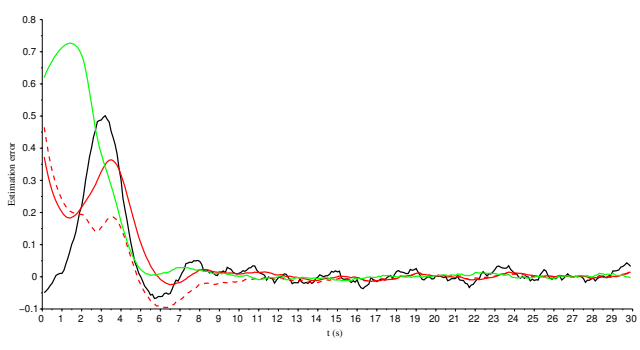

(e) Estimation error for $\mathscr{S}_{\text {min }}$

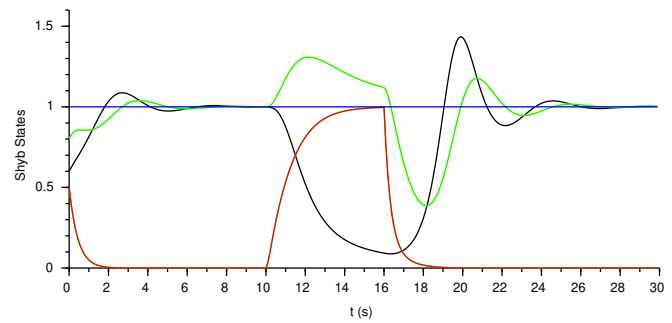

(b) $\mathscr{S}_{h y b}$ states

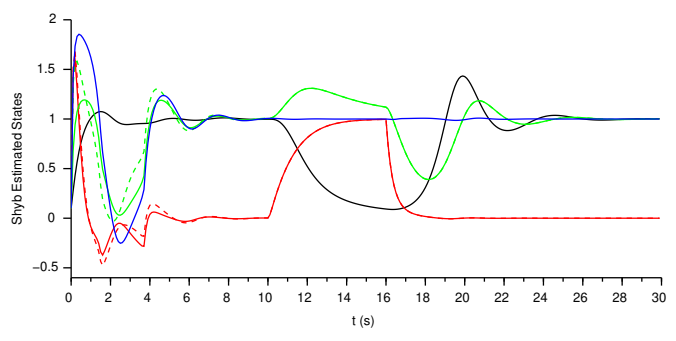

(d) Observer estimation for $\mathscr{S}_{\text {hyb }}$

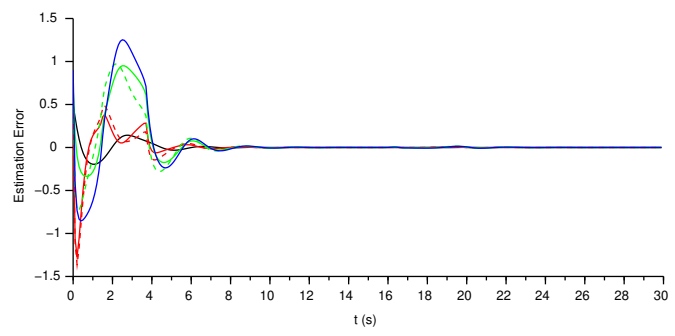

(f) Estimation error for $\mathscr{S}_{h y b}$

Fig. 1. Estimation using the low-power high gain observer in natural coordinates. From top to bottom, on the subfigures: (a)-(b) the states of the physical systems $\mathscr{S}_{\min }\left(\mathscr{S}_{\text {hyb }}\right): x_{1}, x_{2}, x_{3}$ (and $\left.x_{4}\right)$ are depicted in black, red, green and blue solid lines; (c)-(d) state estimation $\hat{x}_{a}^{T}=\left(\hat{x}_{1} \hat{x}_{2} \hat{x}_{2}^{\prime} \ldots \hat{x}_{4}\right)$ is plotted with $\hat{x}_{1}$ in black solid line, $\left(\hat{x}_{2}, \hat{x}_{2}^{\prime}\right)$ in red solid and dash dotted lines, $\left(\hat{x}_{3}, \hat{x}_{3}^{\prime}\right)$ in green solid and dash dotted lines, and $\hat{x}_{4}$ in blue; (e)-(f) the estimation error is drawn using the previous color convention. Due to the low-power high gain observer redundancy, there are two estimates $\tilde{x}_{i}$ and $\tilde{x}_{i}^{\prime}$ for $2 \leq i \leq n-1$.

Simulations are performed using the low-power high gain observer in natural coordinates given by Proposition 8 . The first simulation is led on system $\mathscr{S}_{\min }$ and illustrates the robustness with respect to noise. The $x_{1}$ dynamics and the output $y$ are corrupted by the addition of zero mean Gaussian white noises of standard deviations 0.2 and 0.1 respectively. The former noise is intended to model turbulence and impact of other unmodeled dynamics on the main mode $x_{1}$. System $\mathscr{S}_{\min }$ given by (4) has been simulated with the initial conditions and observer parameters given in Table I. Fictitious outputs required to get a full-ranked Jacobian matrix in Proposition 6 are defined using

$$
\rho: x \mapsto \max \left(0, \varepsilon^{2}-x_{1}^{2}\right)^{2} .
$$

Despite the initial condition $x_{1}(0)=0$, the proposed observer is well defined and exhibits a good convergence property irrespective of the singularity. However, some peaking phenomenon can be noticed at the beginning of the simulation on Figure 1(e) because of the inappropriate choice for initial conditions that "activates" the fictitious outputs. It implies changes in the dynamics of the extension $\tau$, and in turn on the estimates since the observer aims at making $\boldsymbol{\phi}(\hat{\boldsymbol{x}})=\phi_{e}\left(\hat{x}_{a}\right)+\Psi\left(\hat{x}_{a}\right) \tau$ converge, whilst $\phi_{e}\left(\hat{x}_{a}\right)$ is a better representation of the estimates. Then the error asymptotically converges to zero and the role of fictitious outputs vanishes outside the $\hat{x}_{1}>|\varepsilon|$, hence displaying the effectiveness of the proposed observer, particularly with respect to noise filtering.

Simulation parameters for system $\mathscr{S}_{h y b}$ described by (5) are given in Table II. As highlighted in Proposition 1, the system $\mathscr{S}_{\text {hyb }}$ can face an additional observability singularity for $x_{2}+x_{3}+\mu^{\prime}=0$, so we use functions $\rho$ defined by (36) and $\rho^{\prime}(x)=\left(1 /\left(x_{2}+x_{3}+\mu^{\prime}\right)-1 / \varepsilon^{\prime}\right)^{2}$ in order to maintain $\hat{x}_{2}+\hat{x}_{3}+\mu^{\prime}>0$. As it can be noticed on Figure 1(b), the initial dynamics of $\mathscr{S}_{\text {hyb }}$ are driven by the growth rate parameter $x_{4}=\mu$, here assumed to be a constant. $\mathscr{S}_{\text {hyb }}$ behaves as a double oscillator whose main mode amplitude 


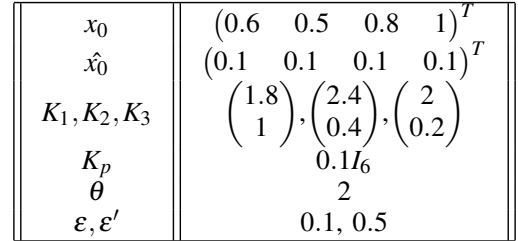

TABLE II

INITIAL CONDITIONS AND PARAMETERS FOR SYSTEM $\mathscr{S}_{h y b}$

$x_{1}$ asymptotically follows a transcritical bifurcation, whilst the enslaved mode amplitude $x_{2}$ is damped thanks to the term $\left(-\mu^{\prime}\right)$. During the time interval $[10 s ; 16 s]$, a control input is applied on the dynamics of the second mode as suggested in [11] in order to excite this oscillator. As expected the coupling -through the shift mode $x_{3}$ in the third equation of (5)- results in an energy flow from the main mode $x_{1}$ to the mode $x_{2}$, forcing $x_{1}$ to go to zero. During the transient phase, Figure 1(d) illustrates the convergence of the state estimates to the real states despite initial values close to zero. As in the first simulation, avoiding the singularity induces some peaking: it is expected since the observer is then built on a system that does not represent exactly the physical model. The estimation tracking is ensured as $x_{1}$ goes to zero due to the control input.

\section{CONCLUSIONS AND FUTURE WORKS}

We have proposed an observer capable of being sufficiently robust with respect to noises and efficient to rebuild the system states while avoiding singularities as shown by the simulation results. The modified low-power high gain observer has provided a solution to many challenges : $i$ ) a rebuild of turbulent wake flow from an unique measured output based on reduced order models, ii) an observability singularity avoidance, iii) a robustness in regard to high frequency noises and sudden changes of the dynamics. It would be interesting to study if the redundancy induced by the low-power high gain observer may be exploited to reduce the proposed observer dimension. The on-going works are on one hand, the experimental validation of the observer in real conditions (the experimental setup is being fully equipped by sensors and actuators) to get a better knowledge of physical phenomena and, on the other hand, the design of feedback laws based on the estimated state to control the flow in order to reduce the drag force. Both perspectives are of great interest for applications in fluid mechanics.

\section{ACKNOWLEDGMENTS}

The authors gratefully acknowledge the financial support from the Region Centre Val de Loire (France) under the project APR-IA COMODO.

\section{REFERENCES}

[1] X. Ma, G.-S. Karamanos, and G. Karniadakis, "Dynamics and lowdimensionality of a turbulent near wake," Journal of fluid mechanics, vol. 410, pp. 29-65, 2000.
[2] J. Östh, B. R. Noack, S. Krajnović, D. Barros, and J. Borée, "On the need for a nonlinear subscale turbulence term in pod models as exemplified for a high-reynolds-number flow over an ahmed body," $J$. Fluid Mech., vol. 747, pp. 518-544, 2014.

[3] S. L. Brunton and B. R. Noack, "Closed-loop turbulence control: Progress and challenges," Appl. Mech. Rev, vol. 67(5), Sept. 2015.

[4] D. Sipp, O. Marquet, P. Meliga, and A. Barbagallo, "Dynamics and control of global instabilities in open-flows: a linearized approach," App. Mech. Rev., vol. 63, no. 3, p. 030801, 2010.

[5] C. W. Rowley and S. T. M. Dawson, "Model reduction for flow analysis and control," Ann. Rev. Fluid Mech., vol. 49, pp. 387-417, 2017.

[6] G. Berkooz, P. Holmes, and J. L. Lumley, "The proper orthogonal decomposition in the analysis of turbulent flows," Annual review of fluid mechanics, vol. 25, no. 1, pp. 539-575, 1993.

[7] C. W. Rowley, I. Mezić, S. Bagheri, P. Schlatter, and D. S. Henningson, "Spectral analysis of nonlinear flows," J. Fluid Mech., vol. 641, pp. 115-127, 2009.

[8] P. J. Schmid, "Dynamic mode decomposition of numerical and experimental data," J. Fluid Mech., vol. 656, pp. 5-28, 2010.

[9] B. R. Noack, K. Afanasiev, M. Morzyński, G. Tadmor, and F. Thiele, "A hierarchy of low-dimensional models for the transient and posttransient cylinder wake," J. Fluid Mech., vol. 497, pp. 335-363, 2003.

[10] G. Tadmor, J. Gonzalez, O. Lehmann, B. Noack, M. Morzynski, and W. Stankiewicz, "Shift modes and transient dynamics in low order, design oriented galerkin models," 45th AIAA Aerospace Sciences Meeting, vol. 2, 2007.

[11] D. M. Luchtenburg, B. Günther, B. R. Noack, R. King, and G. Tadmor, "A generalized mean-field model of the natural and high-frequency actuated flow around a high-lift configuration," Journal of Fluid Mechanics, vol. 623, p. 283316, 2009.

[12] D. Astolfi and L. Marconi, "A high-gain nonlinear observer with limited gain power," IEEE Transactions on Automatic Control, vol. 60, no. 11, pp. 3059-3064, 2015.

[13] P. Bernard, V.Andrieu, and L. Praly, "Expressing an observer in preferred coordinates by transforming an injective immersion into a surjective diffeomorphism," SIAM J. Control Optim., vol. 56, no. 3, pp. 2327-2352, 2018.

[14] J. Gauthier, H. Hammouri, and S. Othman, "A simple observer for nonlinear systems. application to bioreactors." IEEE Transactions on Automatic Control, vol. 37, no. 6, pp. 875-880, 1992.

[15] J. Gauthier and I. Kupka, "Observability and observers for nonlinear systems," SIAM J. Control Optim., vol. 32, no. 4, pp. 975-994, 1994.

[16] F. Deza, E. Busvelle, J. Gauthier, and D. Rakotopara, "High gain estimation for nonlinear systems," Systems \& Control Letters, vol. 18 , no. 4, pp. 295 - 299, 1992. [Online]. Available: http://www.sciencedirect.com/science/article/pii/0167691192900592

[17] P. Bernard, L. Praly, and V. Andrieu, "Tools for observers based on coordinate augmentation," in 2015 54th IEEE Conference on Decision and Control (CDC), 2015, pp. 6324-6329.

[18] V. Andrieu, J.-B. Eytard, and L. Praly, "Dynamic extension without inversion for observers," IEEE International Conference on Decision and Control, pp. $878-883,2014$. 